\title{
Intramyocardial Calcification as a Rare but Significant Indication for Transfemoral TAVI?
}

\author{
Mustafa Gerçek* ${ }^{*}$ [MD] \\ Muhammed Gerçek ${ }^{1}$, [MD] \\ Jan Gummert ${ }^{2}$, [MD] \\ Jochen Börgermann ${ }^{3}$, [MD]
}

\author{
1,Heart and Diabetes Center NRW \\ Clinic for General and Interventional Cardiology/ \\ Angiology \\ 2,Heart and Diabetes Center NRW \\ Clinic for Thoracic and Cardiovascular Surgery \\ 3, Herzzentrum Duisburg \\ Klinik für Herz- und Gefäßchirurgie \\ *Corresponding Author: Mustafa Gerçek, MD \\ Heart and Diabetes Center NRW \\ Clinic for Thoracic and Cardiovascular Surgery \\ Georgstraße 11 \\ 32545 Bad Oeynhausen \\ +49-5731-971331 \\ mustafa.gercek@rwth-aachen.de
}

DOI: https://doi.org/10.32552/2018.ActaMedica.332

Received: 20 September 2018, Accepted: 20 December 2018,

Published online: 25 December 2018

\section{nee ABSTRACT Com}

We report on a 69-year-old female patient with severe valvular aortic stenosis who was hospitalized for cardiac decompensation for aortic valve replacement. Preoperative imaging (X-Ray, cat-scan and CMR) revealed significant and extraordinary intramyocardial calcification pronounced globally in the left ventricle and around the mitral valve. Due to high operative/surgical risk we preferred to approach an aortic valve replacement by transfemoral transcatheter procedure (TAVI-TF). Within the context of an undeniably elevated risk for ventricular injuries and dissections in conventional operations, highly progressed myocardial calcifications should lead to consider and prefer the transfemoral transcatheter aortic valve replacement procedure.

Keywords: Aortic stenosis, intramyocardial calcification, transcatheter aortic valve replacement.

\section{INTRODUCTION}

Conventional aortic valve replacement (AVR) is the gold standard therapy to treat aortic stenosis, the most frequent cardiac valve disease $(1,2)$. The transcetheter transfemoral aortic valve implantation (TAVI-TF) procedure is an ever growing alternative in aortic valve treatment for patients unsuitable for conventional aortic valve replacement $(3,4)$. Intramyocardial calcification is a rare and feared finding in cardiac surgery due to the risk of myocardial rupturing (5-7) and reported in several case reports (8-18). Unfortunately, structural abnormalities like diffuse myocardial calcification are not considered by commonly used risk scores like EuroScore
(19) or STS-Score (20).

We report on a patient with severe aortic stenosis with diffuse myocardial calcification who was not suitable for conventional AVR but could be treated properly by TAVI-TF, forcing to consider TAVI in therapeutic decision making in cases with structural abnormalities and/or high operative risk.

\section{CASE REPORT}

TWe report on a 69-year-old female patient who was admitted to our department of cardiac surgery 
due to cardiac decompensation caused by severe valvular aortic stenosis. The aortic stenosis accompanied by moderate aortic regurgitation had been first diagnosed in this very decompensation with highly calcified tricuspid leaflets, an aortic annulus diameter of $18 \mathrm{~mm}$, an aortic valve area of $0,7 \mathrm{~cm} 2$ and a peak (PPG) and mean pressure gradient (MPG) of $51 \mathrm{mmHg}$ and $32 \mathrm{mmHg}$, respectively. Further transesophageal echocardiographic analysis (TEE) showed normal left ventricular systolic function (61 $\%)$, normal right ventricular systolic function with a dilated right ventricle (ED $=42 \mathrm{~mm})$, highly dilated right and left atrium (ED $=60 \mathrm{~mm}$, both) and no signs of left ventricular outflow tract obstructions. TEE also presented mixed mitral valve disease with moderate stenosis (mean gradient $10 \mathrm{mmHg}$, valve area $1,0 \mathrm{~cm}^{2}$ ) and moderate to severe eccentric regurgitation due to severely calcified leaflets with stiffness of the posterior mitral leaflet (PML). In addition, the tricuspid valve showed a moderate to severe regurgitation with an estimated right systolic pulmonary artery pressure of $65 \mathrm{mmHg}$. Despite phenprocoumon medication (INR 2 to 3 ) to avoid atrial fibrillation caused embolic events, a thrombus in the left atrial appendage was detected by TEE.

Right heart catheterization revealed secondary post-capillary pulmonary hypertension with a mean pulmonary artery pressure of $52 \mathrm{mmHg}$ and a mean pulmonary capillary wedge pressure (PCWP) of $35 \mathrm{mmHg}$. Filling pressures of the right heart were significantly elevated (right ventricular end-diastolic pressure $15 \mathrm{mmHg}$, mean right atrial pressure 20 with a v-wave of $20 \mathrm{mmHg}$ ).

Furthermore, the patient presented a left bundle branch block (LBBB) and persistent atrial fibrillation (AF) causing tachyarrhythmia absoluta (TAA), chronic renal failure (eGFR $48 \mathrm{ml} / \mathrm{min}$ ) and essential arterial hypertension with hypertensive heart disease.

In addition, the patient reported about an ischemic stroke without residual neurological symptoms 7 years ago, a temporary retinal artery occlusion 8 years ago and a tuberculosis infection some 40 years ago. Cardiovascular risk factors were - in addition to arterial hypertension - hyperlipoproteinemia and moderate obesity. Cardiac catheterization showed no complex coronary disease. She was classified as NYHA IV and CCS II. The mean logistic EUROSCORE was 5,8\%, the EUROSCORE II was $4,14 \%$ and the STS-Score was 2,07 \%.

Preoperative $x$-ray imaging of the thorax was highly suspicious for diffuse myocardial calcification. It showed a hypertrophied calcified heart in a kyphotic chest and a scoliosis of the thoracic spine
(Figure 1a).

Computerized tomography and angiography of the aorta and the heart that were performed to verify and examine the findings, revealed a commissural calcified aortic valve and a severely calcified mitral valve, without significant calcification or stenosis of the coronary arteries. The left ventricle was comprehensively calcified. (Figure 1b-e). Besides the thrombus $(17 \times 10 \mathrm{~mm})$ in left atrial appendage was confirmed. The iliac and femoral artery showed no significant stenosis, with a common femoral artery diameter of $8 \mathrm{~mm}$ on both sides, hence transfemoral procedures were feasible. Additionally, the cat scan further showed apical subpleural lesions on both sides of the lung, possibly associated with the tuberculosis infection from 1970.

To further analyze the disease related alterations cardiovascular magnetic resonance imaging (CMR) was performed presenting normal ventricular dimensions but hypertrophied and hypokinetic basal parts of the left ventricular myocardium. The intramurally located calcifications were diffused to the left ventricle with apical, subepicardial and pericardial calcified areas. In addition to the calcification, the CMR showed diffuse fibrotic lesions of the left ventricular myocardium.

To conclude, the patient showed a multiple valve disease with leading low-flow-low-gradient aortic stenosis with preserved ejection fraction, which may be caused by the concomitant mitral regurgitation, atrial fibrillation and paradox or restrictive ventricular filling because of the intramyocardial calcification.

Due to these findings, the comorbidities, the significant intramyocardial calcification and fibrotic lesions we decided to treat the aortic stenosis with transfemoral transcatheter aortic valve implantation (TAVI-TF). We were further reinforced in our decision by the fact that myocardial calcification is associated with an elevated risk for ventricular/atrial injuries and dissections in conventional surgical procedures (5-7).

To provide hemodynamics and rhythm control during the procedure a temporary pacemaker was placed on the patient. The aortic annulus was illustrated by a pigtail-catheter which was then changed to a Stiffwire to load and introduce a 26 mm Edwards SAPIEN 3 Transcatheter Heart Valve prosthesis. The prosthesis was put in place safely and released under rapid pacing. Angiographic control of the implanted prosthesis showed no relevant aortic regurgitation and an effectively reduced MPG from $26 \mathrm{mmHg}$ to $0 \mathrm{mmHg}$.

Afterwards the patient was shifted to the intensive care unit for three days without complications. In 
standard care further mobilization was successfully performed by physiotherapist. Five days post-surgery the patient was discharged in good general condition without her wounds showing signs of irritation and still remaining mitral and tricuspid valve disease. Oral anticoagulation with phenprocoumon to maintain an INR between 2 and 3 was applied and recommended to avoid embolic events after valve implantation and with respect to the persistent atrial fibrillation.

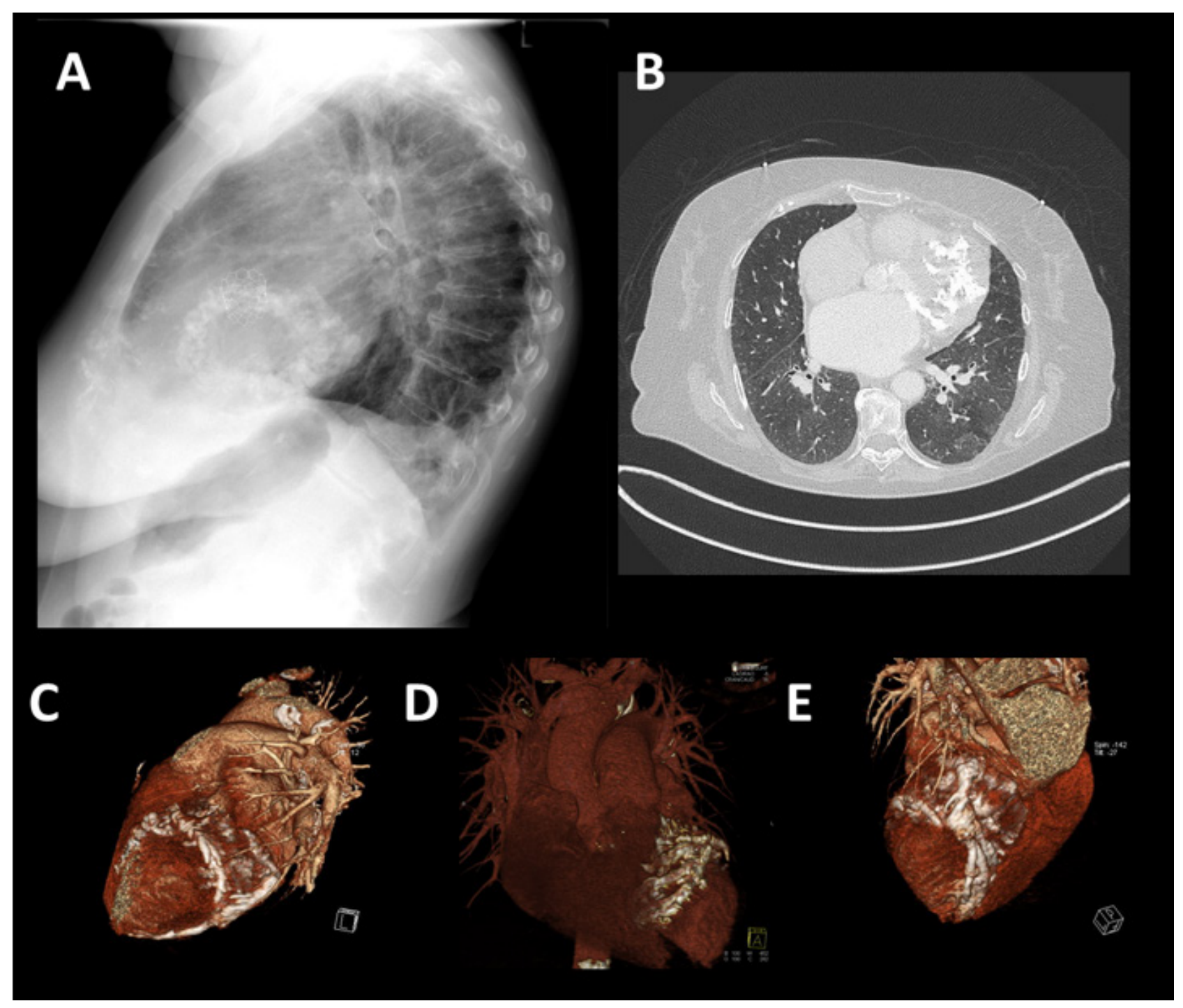

Figure 1 Preoperative imaging ( $\mathrm{x}$-ray and cat scan) revealed massive calcification of the left ventricle

\section{DISCUSSION}

Diffuse myocardial calcification was reported in several cases and were associated with various systemic diseases like sepsis $(14,18)$, chronic renal failure $(10-13,15-17)$ or tuberculosis infection $(8,9)$. A detailed characterization in terms of pathophysiology or progress due to mostly hidden and asymptomatic development of the calcification is still missing. Endomyocardial fibrosis (EMF) a rarely understood disease presenting with fibrotic alterations and lesions in the myocardium (21) that are highly suspected to cause calcification in disease progression $(22,23)$ may be associated to the reported case that shows diffuse fibrotic lesions alongside the dominant calcified areas.

Myocardial calcification is a feared finding in cardiac surgery, especially in mitral valve surgery, due to the danger of myocardial rupture or dissection (57). In spite of this evidence-based guidelines or recommendations for dealing with this type of calcification do not exist. Not only to prevent patients from adverse events but also to treat valve diseases causing heart insufficiency, peripheral and pulmonary edema and consecutively having a crucial impact on life quality a non-touch technique is 
needed. The TAVI procedure shows acceptable results for patients with high risk and promising results for patients with intermediate risk profile (24, 25). Therefore from our point of view, this technique should be at least more commonly considered in patients with severe intramyocardial calcification and a high perioperative risk rather than risking a conventional operation or denying operative care of the patient altogether.

Fortunately, the patient of this case report recovered rapidly despite remaining combined mitral and tricuspid valve disease emphasizing the benefit of the afterload reduction in patients with multiple valve diseases.

\section{CONCLUSION}

We report on a rare case of globally diffused massive intramyocardial calcification accompanied by severe aortic stenosis. In times of evermore standardized and established protocols of transfemoral transcatheter procedures and with respect to the feared complications of myocardial dissection or rupture this case is representing a forcing criterion and should lead to favor the TAVI procedure over conventional surgery.

\section{we REFERENCES Cem}

[1] Baumgartner H, Falk V, Bax JJ, et al. 2017 ESC/EACTS Guidelines for the management of valvular heart disease. European Heart Journal 2017; 38(36): 2739-91.

[2] Nishimura RA, Otto CM, Bonow RO, et al. 2017 AHA/ACC Focused Update of the 2014 AHA/ACC Guideline for the Management of Patients With Valvular Heart Disease: A Report of the American College of Cardiology/American Heart Association Task Force on Clinical Practice Guidelines. Circulation 2017; 135(25): e1159-e95.

[3] Leon MB, Smith CR, Mack M, et al. Transcatheter Aortic-Valve Implantation for Aortic Stenosis in Patients Who Cannot Undergo Surgery. New England Journal of Medicine 2010; 363(17): 1597-607.

[4] Leon MB, Smith CR, Mack MJ, et al. Transcatheter or Surgical Aortic-Valve Replacement in Intermediate-Risk Patients. New England Journal of Medicine 2016; 374(17): 1609-20.

[5] Karlson KJ, Ashraf MM, Berger RL. Rupture of left ventricle following mitral valve replacement. The Annals of thoracic surgery 1988; 46(5): 590-7.

[6] Kim KW, Kim JH, Park SH, et al. Left atrial wall dissection after mitral valve replacement. Journal of cardiovascular ultrasound 2013; 21(3): 145-7.

[7] Schmid ER, Schmidlin D, Jenni R. Images in cardiology. Left atrial dissection after mitral valve reconstruction heart 1997; 78(5): 492.

[8] Butz T, van Bracht M, Meissner A, et al. Mitral valve disease as well as uncommon extensive epipericardial and intramyocardial calcification secondary to massive mitral annular calcification. Eur J Echocardiogr 2010; 11(1): 85-8.

[9] D'Angelo C, Zagnoni S, Riva L, et al. Idiopathic massive myocardial calcification: a rare cause of heart failure. G Ital Cardiol (Rome) 2016; 17(2): 91-4.

[10] Henderson RR, Santiago LM, Spring DA, et al. Metastatic myocardial calcification in chronic renal failure presenting as atrioventricular block. N Engl J Med 1971; 284(22): 1252-3.

[11] Illyes G, Taraba I. Myocardial calcification in chronic renal failure. Orv Hetil 1989; 130(17): 883-6.

[12] Martin-Cuartero J, Yague-Romeo D, Abril-Avellanas E. Myocardial calcification in chronic renal failure. Rev Esp Cardiol 2007; 60(10): 1092.
[13] Rahman M, Kim SJ, Kim JS, et al. Myocardial calcification and hypertension following chronic renal failure and ameliorative effects of furosemide and captopril. Cardiology 2010; 116(3): 194-205.

[14] Rossi MA, Santos CS. Sepsis-related microvascular myocardial damage with giant cell inflammation and calcification. Virchows Arch 2003; 443(1): 87-92.

[15] Rostand SG, Sanders C, Kirk KA, et al. Myocardial calcification and cardiac dysfunction in chronic renal failure. Am J Med 988; 85(5): 651-7.

[16] Shackley BS, Nguyen TP, Shivkumar K, et al. Idiopathic massive myocardial calcification: a case report and review of the literature. Cardiovasc Pathol 2011; 20(2): e79-83.

[17] Shibuya A, Sugimoto T, Yamakado M, et al. A case of chronic renal failure with diffuse hepatic and myocardial calcification. Nihon Naika Gakkai Zasshi 1985; 74(9): 1257-64.

[18] Van Kruijsdijk RC, van der Heijden JJ, Uijlings R, et al. Sepsisrelated myocardial calcification. Circ Heart Fail 2011; 4(5): e16-8.

[19] Nashef SA, Roques F, Michel P, et al. European system for cardiac operative risk evaluation (EuroSCORE). European journal of cardio-thoracic surgery : official journal of the European Association for Cardio-thoracic Surgery 1999; 16(1): 9-13.

[20] TSoT S. Executive Summary: Society of Thoracic Surgeons Spring 2007 Report. Chicago: The Society of Thoracic Surgeons 2007

[21] Mocumbi AO. Endomyocardial fibrosis: A form of endemic restrictive cardiomyopathy. Global cardiology science \& practice 2012; 2012(1): 11.

[22] Somers K, Williams AW. Intracardiac calcification in endomyocardial fibrosis. British Heart Journal 1962; 24: 324-8.

[23] Bhatti K, Lopez-Mattei J. Endomyocardial Fibrosis. StatPearls. Treasure Island (FL) 2018.

[24] Leon MB, Smith CR, Mack M, et al. Transcatheter aortic-valve implantation for aortic stenosis in patients who cannot undergo surgery. N Engl J Med 2010; 363(17): 1597-607.

[25] Leon MB, Smith CR, Mack MJ, et al. Transcatheter or Surgical Aortic-Valve Replacement in Intermediate-Risk Patients. N Engl J Med 2016; 374(17): 1609-20. 